\title{
Extraordinary optical transmission through nonlocal holey metal films
}

\author{
David, Christin; Christensen, Johan
}

Published in:

Applied Physics Letters

Link to article, DOI:

$10.1063 / 1.4991016$

Publication date:

2017

Document Version

Publisher's PDF, also known as Version of record

Link back to DTU Orbit

Citation (APA):

David, C., \& Christensen, J. (2017). Extraordinary optical transmission through nonlocal holey metal films. Applied Physics Letters, 110(26), [261110]. https://doi.org/10.1063/1.4991016

\section{General rights}

Copyright and moral rights for the publications made accessible in the public portal are retained by the authors and/or other copyright owners and it is a condition of accessing publications that users recognise and abide by the legal requirements associated with these rights.

- Users may download and print one copy of any publication from the public portal for the purpose of private study or research.

- You may not further distribute the material or use it for any profit-making activity or commercial gain

- You may freely distribute the URL identifying the publication in the public portal

If you believe that this document breaches copyright please contact us providing details, and we will remove access to the work immediately and investigate your claim. 


\section{Extraordinary optical transmission through nonlocal holey metal films}

Christin David and Johan Christensen

Citation: Appl. Phys. Lett. 110, 261110 (2017); doi: 10.1063/1.4991016

View online: http://dx.doi.org/10.1063/1.4991016

View Table of Contents: http://aip.scitation.org/toc/apl/110/26

Published by the American Institute of Physics

\section{Articles you may be interested in}

As-grown two-dimensional $\mathrm{MoS}_{2}$ based photodetectors with naturally formed contacts

Applied Physics Letters 110, 261109 (2017); 10.1063/1.4990968

Electrode modulated capacitance-electric field nonlinearity in metal-insulator-metal capacitors

Applied Physics Letters 110, 263503 (2017); 10.1063/1.4989531

Laguerre-Gaussian quasi-modal q-plates from nanostructured glasses

Applied Physics Letters 110, 261108 (2017); 10.1063/1.4990954

Electrical switching of the magnetic vortex circulation in artificial multiferroic structure of $\mathrm{Co} / \mathrm{Cu} / \mathrm{PMN}-\mathrm{PT}(011)$

Applied Physics Letters 110, 262405 (2017); 10.1063/1.4990987

Cooperative effects in an ensemble of planar meta-atoms

Applied Physics Letters 110, 261101 (2017); 10.1063/1.4989691

A highly efficient element for generating elliptic perfect optical vortices

Applied Physics Letters 110, 261102 (2017); 10.1063/1.4990394

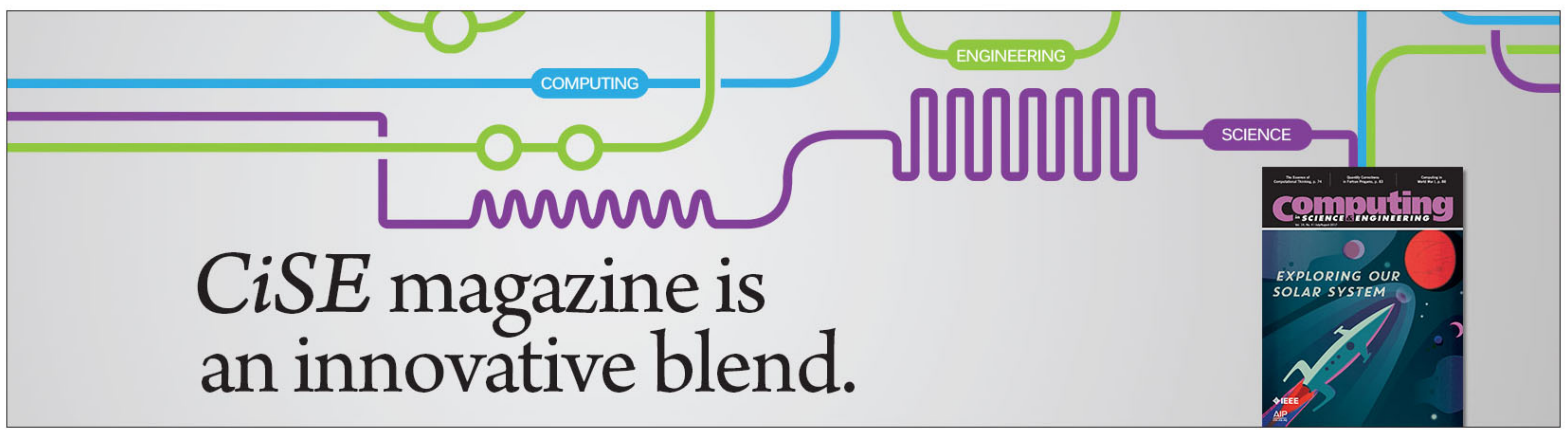




\title{
Extraordinary optical transmission through nonlocal holey metal films
}

\author{
Christin David ${ }^{1, a)}$ and Johan Christensen ${ }^{2}$ \\ ${ }^{1}$ DTU Fotonik, Technical University of Denmark, 2800 Kongens Lyngby, Denmark \\ ${ }^{2}$ Instituto Gregorio Millán Barbany, Universidad Carlos III, 28916 Leganés, Spain
}

(Received 15 May 2017; accepted 19 June 2017; published online 30 June 2017)

\begin{abstract}
We investigate nonlocal electrodynamics based on the generalized hydrodynamic approach including electron diffusion in holey gold films, showing extraordinary optical transmission (EOT). Dramatic changes with respect to the local approximation for rather large film thicknesses $t \lesssim 100 \mathrm{~nm}$ impact both reflectance and absorbance at normal incidence. Beyond the familiar resonance blueshift with the decreasing film thickness, the interference of longitudinal pressure waves in the holey structure generates an unexpected oscillatory response with geometrical parameters. An increase in the transmittance relative to the local result of up to $33 \%$ (typically up to $\pm 6 \%$ at resonance) was observed, but it can also be suppressed when absorption dominates. We expect that our findings will stimulate additional studies of EOT when taking into consideration nonlocal effects in holey films of experimentally accessible parameters in two-dimensional hole arrays. Published by AIP Publishing. [http://dx.doi.org/10.1063/1.4991016]
\end{abstract}

The ability to efficiently squeeze light through a metal film with an array of holes smaller than the wavelength of the incoming light has fascinated and inspired science and technology for two decades. ${ }^{1-10}$ Areas such as not only sensing and spectroscopy ${ }^{11}$ but also the design of optical filters, ${ }^{12}$ plasmonic colors, ${ }^{13}$ lasers, ${ }^{14}$ and quantum plasmonics ${ }^{15}$ benefit from the extreme confinement and sensitivity of the transmittance with the geometrical configuration of hole arrays in metal films and illumination conditions. ${ }^{710}$ This extraordinary optical transmission (EOT) originates in the coupling of light to surface excitations unique to metal systems: plasmons.

Light transmitted through tiny apertures can be significantly enhanced thanks to the excitation of surface plasmons on and through the patterned structure. ${ }^{16}$ The resonance of this phenomenon is strongly influenced by the environment including the presence of molecules, which makes label-free biosensing an important application for hole arrays. The advantages lie in the straightforward optical detection at normal incidence, integration in microfluidic devices, ${ }^{17}$ and miniaturization, allowing a small sensor size and on-chip, multiplexing technology with high sensitivity and low detection limits. ${ }^{18}$ In particular, gold and silver in air or water were intensively studied with hole sizes between 100 and $500 \mathrm{~nm}$ and lattice periods of $400-1000 \mathrm{~nm}$ for results in the visible and near infrared. ${ }^{19}$ Advancing the rich palette of applications and unraveling physical mechanisms require progress in the electromagnetic treatment of holey metal films. When the conduction band electrons in metals are strongly confined, their quantum nature becomes important. Short-ranged electron-electron interaction effects were investigated mainly in the limit of isolated particles or dimers in the experiment. ${ }^{20-24}$ The limitations of ab initio methods ${ }^{25-29}$ to small particle systems have sparked the interest in teaming up classical electrodynamics, i.e., the local response approximation (LRA), with a dynamic description of the free

a)chrida@fotonik.dtu.dk electron gas. In the hydrodynamic framework, the (linearized) Navier-Stokes equation (1) is coupled to the electromagnetic wave equation, separating the dynamics of bound, core electrons, and the free conduction band electrons. This yields additional longitudinal fields stemming from the electron plasma motion ${ }^{30-33}$ subject to the Coulomb interaction (given by a Thomas-Fermi pressure term) and electron diffusion.

These pressure waves were studied for isolated particles $^{23,30,34,35}$ and planar structures ${ }^{36,37}$ and in nonlocal gratings and hyperbolic materials. ${ }^{38-42}$ In brief, nonlocal modes typically yield (i) a correction to the plasmon resonance condition, resulting in blueshifts with respect to the LRA due to an effective reduction of the permittivity for increasingly small system sizes and (ii) plasmon broadening due to the introduction of further decay channels. Their coupling strength depends on the Fermi velocity $v_{F}$, while the coupling itself is determined via an additional boundary condition that prohibits electron transfer into the dielectric surroundings. 32,33

In this letter, we perform extensive parameter studies to investigate the impact of nonlocal electron dynamics in holey metal films and their EOT performance. This is based on the theoretical framework for the nonlocal optical response (NOR) in two-dimensional structures previously established, ${ }^{33}$ extended to include electron diffusion, which provides a useful tool where full numerical calculations are tedious to carry out. Free standing holey gold films of thickness $t$, lattice constant $a$, and hole radius $0 \leq r \leq 0.5 a$ are considered as illustrated in Fig. 1. The material is described with tabulated experimental data, ${ }^{43}$ and the structure is illuminated at normal incidence. For homogeneous metal films, no nonlocal response arises at normal incidence since additional longitudinal modes are not excited for vanishing $k_{\|} \equiv 0$. However, anomalous diffraction in the hole array provides finite components of the in-plane wave vector. ${ }^{33}$ Interestingly, nonlocal modes are excited at surprisingly large structural parameters with a clear influence on the optical 


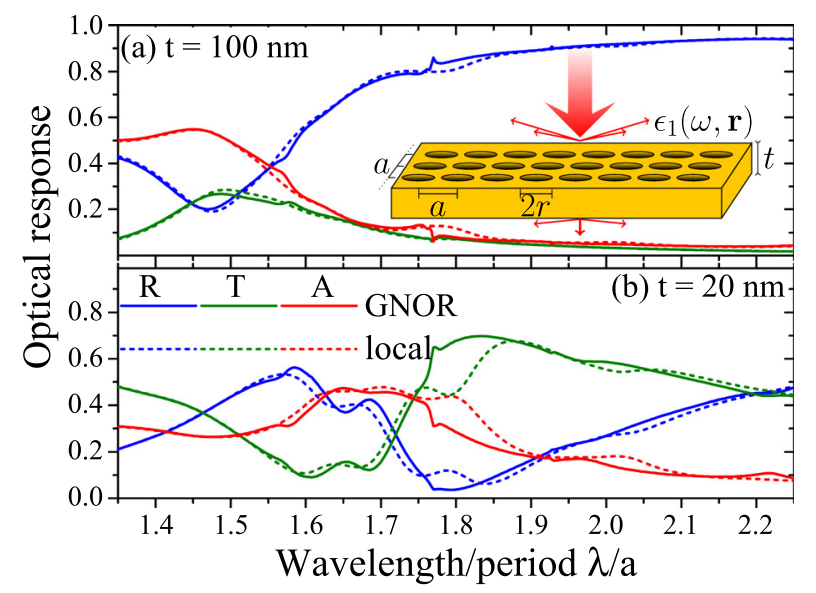

FIG. 1. Transmittance $T$ (green), reflectance $R$ (blue), and absorbance $A$ (red) for patterned gold ${ }^{43}$ films comprised of circular air holes in a square array with period $a=400 \mathrm{~nm}$ and radius $r=0.25 a$ suspended in air, illuminated at normal incidence (see the inset; $k_{\|}=0$ throughout this work). We compare the local response approximation (dashed lines) with the generalized nonlocal optical response (GNOR, solid lines). The film has thicknesses of (a) $t=100 \mathrm{~nm}$ and (b) $t=20 \mathrm{~nm}$.

response of the system. The study of large-scale, two-dimensional setups allows a different approach to the detection and verification of nonlocal phenomena at experimentally accessible geometrical parameters, including rectangular structures such as checkerboards. ${ }^{49}$

We employ the rigorous coupled wave analysis (RCWA) to describe the modulated, two-dimensional film..$^{33,44,45}$ The in-plane momentum $\overrightarrow{k_{\|}}$can provide finite values stemming from diffraction into higher order waves. The transmittance of the periodic structure is obtained combining RCWA with the scattering matrix theory for the finite slab commonly used for photonic crystal structures. Nonlocal (longitudinal) solutions of the electromagnetic field are computed with an eigenvalue equation obtained from the hydrodynamic equation. ${ }^{46}$ More details are given in the supplementary material.

We discuss the nonlocal optical response in holey gold films comparing two models based on the hydrodynamic approach, where the induced current density $\vec{j}^{\text {ind }}$

$$
\vec{j}^{\text {ind }}=\frac{i}{\omega+i \gamma_{p}}\left(\frac{\omega_{p}^{2}}{4 \pi} \vec{E}-\left(\beta^{2}+D\left(\gamma_{p}-i \omega\right)\right) \nabla \rho^{\text {ind }}\right)
$$

is related to the induced charge density $\rho^{\text {ind }}$ through the continuity equation $\nabla \vec{j}^{\text {ind }}=i \omega \rho^{\text {ind }}$. The wave number is $k=\omega / c$ with $c$ being the speed of light in vacuum. The properties of the material $(\mathrm{Au})$ are captured in the plasmon frequency $\omega_{p}$ $(=9.0 \mathrm{eV})$ and damping $\gamma_{p}(=0.071 \mathrm{eV})$, which reflects the lifetime of the plasmon excitation, as well as in the dielectric background $\varepsilon_{b}(=9.0)$ and the interaction strength of the nonlocal electrons $\beta=\sqrt{3 / 5} v_{F}^{2}\left(v_{F} \cong 1.4 \times 10^{6} \mathrm{~m} / \mathrm{s}\right) .{ }^{47}$

It was shown previously ${ }^{32,48}$ that the nonlocal strength $\beta$ of the hydrodynamic approach can be extended to include diffusion phenomena in an effectively complex $\beta$-parameter $\beta_{G N O R}^{2}=\beta^{2}+D\left(\gamma_{p}-i \omega\right)$, coined as the generalized nonlocal optical response (GNOR). This remains true for the matrix-valued description within the RCWA. ${ }^{33}$ The real part of the nonlocal coupling strength is thus increased, which has so far only included the electron-electron (Coulomb) interaction. This leads to an enhanced blueshift in the plasmon resonance with respect to the LRA. Furthermore, it introduces an imaginary part and thus an additional damping associated with electron diffusion. This adds to plasmon broadening.

Here, we discuss the two extremes of vanishing diffusion $\left(D \equiv 0 \Rightarrow \beta_{G N O R}^{2}=\beta^{2}\right)$ and the bulk value $D=v_{F}^{2} \gamma_{p}$ $\approx 14 v_{F}^{2} \neq 0$, following from the classical gas theory for an electron plasma (obeying Fermi statistics). This also reflects the mean free path $v_{F} \gamma_{p}$ of the conduction band electrons. We believe that this is an appropriate choice for the planar geometry studied here, representing an impact from the bulk metal material, in contrast to the spherical nanoparticles studied in Ref. 48, where a diffusion parameter smaller by an order of magnitude was deduced. We discuss later on some intermediate values of the diffusion parameter. Note that with a complex $\beta$, similar numerical difficulties arise as for the complex permittivity $\epsilon$ describing metal materials, ${ }^{44,45}$ and particular care has to be taken with a view to the numerical method. Technical details concerning the convergence of the RCWA extended by nonlocal contributions can be found in the supplementary material and Ref. 33.

We compute two examples and plot the spectra of optical coefficients in Fig. 1 where we concentrate on the optical response beyond $\lambda / a>1$ where transmission through the subwavelength air holes is enhanced. At the larger film thickness (Fig. 1(a)), the differences between the local response approximation (LRA) and the generalized nonlocal optical response (GNOR) are already visible though subtle. Mainly, we observe an increase in the reflectance where the incoming light is reflected at the nonlocal modes. The emergence of an additional absorbance peak at $\lambda / a=1.57$ and of a Fano-type resonance at $\lambda / a=1.77$ is the further indication of an impact of nonlocal effects on the optical response that goes beyond typically found resonance shifts and plasmon broadening. In Fig. 1(b), the film thickness is reduced to $t=20 \mathrm{~nm}$, and while the overall peak positions are more strongly shifted, the intriguing result here is the reduction in reflectance that yields an increase in the observed transmittivity. At the resonance position $\lambda / a=1.83$ (shifted by $16.5 \mathrm{~nm}$ ), the transmittivity is increased by $3 \%$. Comparing the transmittivity at $\lambda / a=1.8$, we observe an increase of $33 \%$ due to strong broadening. In turn, the absorbance of the system is also reduced. This means that the presence of the additional longitudinal waves provides an additional efficient coupling mechanism to add up to the extraordinary transmission instead of absorbing the incoming light.

In Fig. 2, we investigate the emergence of nonlocal phenomena with the film thickness for an intermediate hole radius $r=0.25 a$ and two different hole separations. This structural parameter has a clear analogue to the case of a homogeneous film. ${ }^{36}$ As expected, the influence of spatial dispersion decreases with the increasing slab thickness $t$. Interestingly, the smaller lattice period of $a=100 \mathrm{~nm}$, shown in Fig. 2(a), does not exhibit as dramatic effects as the larger value of $a=400 \mathrm{~nm}$, shown in Fig. 2(b). It should be noted, however, that the hole radius was scaled with the period. Here, still at a film thickness of $t=100 \mathrm{~nm}$, a sizeable blueshift of the transmittance resonance position is obtained. 

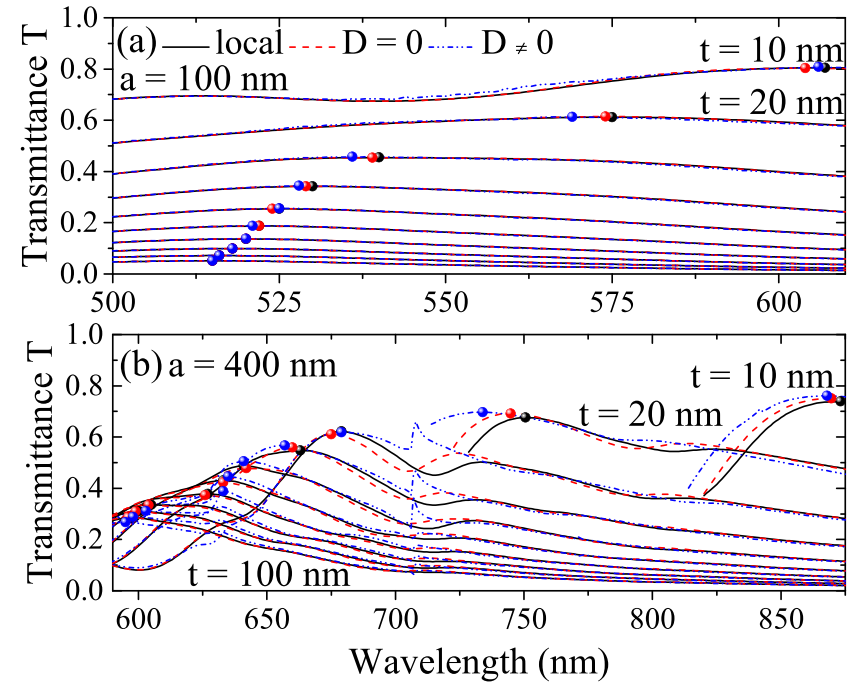

FIG. 2. Comparing transmittance spectra with varying film thicknesses (10, $\ldots, 100 \mathrm{~nm}$ ) for classical local (solid, black) calculations with NOR (diffusion constant $D=0$, red dashed) and GNOR $\left(D=v_{F}^{2} / \gamma_{p}\right.$, blue dotted) for $r=0.25 a$ and (a) $a=100 \mathrm{~nm}$ and (b) $a=400 \mathrm{~nm}$. The resonance positions are highlighted with color-coded dots for convenience.

The GNOR theory yields even stronger blueshifts than the case of vanishing diffusion when compared to the LRA. This is related to a larger real part in $\beta_{\mathrm{GNOR}}$ and an enhanced plasmon broadening connected to its imaginary part. We emphasize that increasing the lattice period (while scaling the radius as $r / a$ ) enhances the impact of nonlocal effects due to the reduction in the lowest order reciprocal vector $\vec{G}=2 \pi / a$. Diffraction becomes more important where the wavelength $\lambda(k=2 \pi / \lambda)$ is of the same magnitude as the structural parameter $a$.

In order to maintain a high impact of nonlocal modes, the hole radius needs to be relatively large, which we study in Fig. 3. Again, a blueshift of the transmittance and a broadening are observed in the spectra obtained from the nonlocal theory in Fig. 3(a) when the size of the air holes is increased. A closer look at Figs. 3(b) and 3(c) reveals that, as expected, the GNOR approach yields stronger blueshifts of the main transmittance peak and overall impacts the optical response of the metal holey film at structural parameters closer to the local limit. Interestingly, both nonlocal theories exhibit an oscillatory behavior with the hole size. This

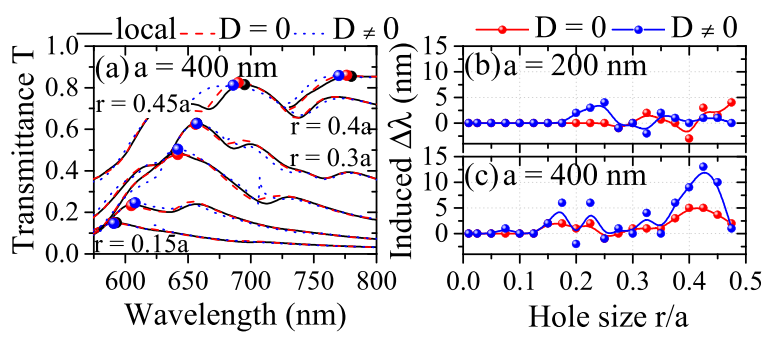

FIG. 3. Comparing transmittance spectra with varying hole radii $r / a$ $(0.15, \ldots, 0.45)$ for classical local (solid black) calculations with NOR (diffusion constant $D=0$, red dashed) and GNOR $\left(D=v_{F}^{2} / \gamma_{p}\right.$, blue dotted) for $t=50 \mathrm{~nm}$ and (a) $a=400 \mathrm{~nm}$. The resonance position is marked with a dot for convenience. (b) and (c) The resonance shift as a function of the hole size $r / a$ is shown for lattice periods of (b) $a=200 \mathrm{~nm}$ and (c) $a=400 \mathrm{~nm}$. The data points are connected by a spline to stress the observed oscillatory behavior. indicates that the longitudinal, nonlocal mode excited in the crystal structure is subject to interference effects that allow canceling the observed phenomena arising from additional electron dynamics. Transversal modes are governed by the wave number $k_{z}^{\vec{G}}=\sqrt{(\omega / c)^{2} \epsilon_{\perp}-\left|\vec{k}_{\|}-\vec{G}\right|^{2}}$, while longitudinal modes obey $q_{z}^{\vec{G}}=\sqrt{\epsilon_{\perp} /\left(\beta^{2} \epsilon_{b}\right) \omega(\omega+i \gamma)-\left|\overrightarrow{k_{\|}}-\vec{G}\right|^{2}}$. The resulting wave numbers (inverse mode wavelengths) show a similar spectral structure. However, $q_{z} / k_{z} \sim 10 \ldots$ $\sqrt{(c / \beta)^{2} / \epsilon_{b}} \approx 90$, indicating that nonlocal modes form standing waves in metal bridges between air holes with a nodal number higher than transversal waves by an order of magnitude, which makes them very sensitive to small changes in the geometrical structure.

We compare transmittance spectra for varying hole separations $a$ (while fixing the radius at $r / a$ ) for classical local calculations with the nonlocal optical response models in Fig. 4. The most dramatic effects are observed for the smallest film thickness (Fig. 4(a)) and the largest lattice period in accordance with the previously discussed results. Next, we study how nonlocality influences the transmittance. We consider several values for the electron diffusion constant from the standard hydrodynamic model $(D=0)$ to the bulk model $\left(D=v_{F}^{2} / \gamma_{p}\right)$ in Fig. 5(a) and compare the relative change in the transmittance calculated as $\left(1-T_{\mathrm{loc}} / T_{\mathrm{nl}}\right)$ in $\%$. All cases of the nonlocal strength parameter behave similarly. However, it is surprising that both a reduction and an increase in the transmittance are observed, here as a function of the film thickness, with very steep transitions between these cases. This relative change in transmittance lies between $-4 \%$ and $+6 \%$ in the considered cases compared to the classical local result and increases, e.g., when the lattice period is further increased. Figures 5(b) and 5(c) show how the increase or decrease in the transmittance translates into an influence on both the reflectance and absorbance for two selected cases. In Fig. 5(b), the increase in transmittancetogether with the appearance of an additional resonance-in the nonlocal case is accompanied by a reduction in both reflection on the front surface and absorbance, thus leading to an efficient transmittance through the $t=10 \mathrm{~nm}$ thick film. In addition, a pronounced absorption peak appears at a larger wavelength, leading to the strong reduction in transmittance between the also classically observed resonances.

On the other hand, the transmittance is reduced in the $t=20 \mathrm{~nm}$ thick slab in Fig. 5(c) due to reflection at a resonant

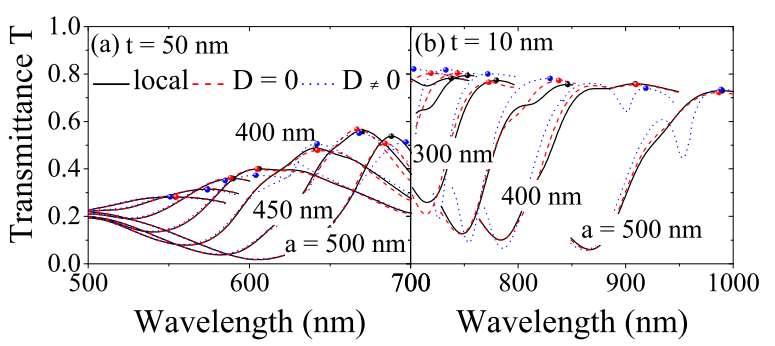

FIG. 4. (a) and (b) Comparing transmittance spectra with varying hole separations $a(200, \ldots, 500 \mathrm{~nm})$ for classical local (black solid) calculations with NOR (diffusion constant $D=0$, red dashed) and GNOR $\left(D=v_{F}^{2} / \gamma_{p}\right.$, blue dotted) for $r=0.25 a$ and (a) $t=50 \mathrm{~nm}$ and (b) $t=10 \mathrm{~nm}$. The resonance position is marked with a dot for convenience. 


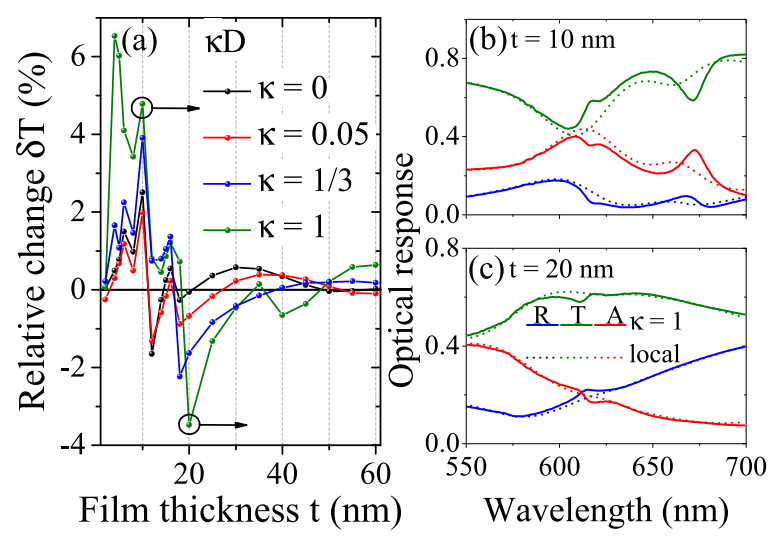

FIG. 5. (a) Change in transmittance with $t$ for diffusion constants $D=\kappa v_{F}^{2} / \gamma_{p}$, period $a=200 \mathrm{~nm}$, and hole size $r=0.25 a$. (b) and (c) Spectra (transmittance $\mathrm{T}$, green; reflectance R, blue; absorbance A, red) corresponding to the geometries marked with a circle in (a) for (b) $t=10 \mathrm{~nm}$ and (c) $t=20 \mathrm{~nm}$

front surface mode, which in turn reduces the share absorbed inside the film. It is such additional reflective or absorptive modes that might allow for experimental verification of the described effects. Increasing the parallel momentum of the incoming light enhances the impact of nonlocal phenomena. ${ }^{33}$ However, varying the angle of incidence within the first Brillouin zone $k_{\|}=0\left(0^{\circ}\right)$ and $k_{\|}= \pm \pi / a\left(90^{\circ}\right)$ does not dramatically increase the parallel momentum and therefore does not alter the observations significantly (see the supplementary material).

Finally, we show an example for the distribution of the nonlocal field (inside the holey metal film, underneath the front surface) in Fig. 6(a) at the resonance wavelength of the nonlocal response using the standard hydrodynamic model ( $D=0, \lambda=719 \mathrm{~nm})$. A strong dipolar mode is observed in the corresponding induced charge density $\rho^{\text {ind }}$ (see Fig. 6(b)). Note that the non-vanishing contribution to the electron density inside the air holes in these real space calculations is due to the early truncation of the Fourier series. The finite number of modes included in this calculation (restricted to $N=1849$ plane waves) is restricted by computational power.

In summary, we have studied the impact of nonlocal, longitudinal electron waves on the optical properties on EOT phenomena in metal films with circular holes. Our method is applicable also to rectangular unit cells where plasmonic crystals are studied in checkerboard configurations. ${ }^{49} \mathrm{We}$ discussed the electron diffusion added in the GNOR theory, which leads to an effectively complex $\beta$-parameter. This

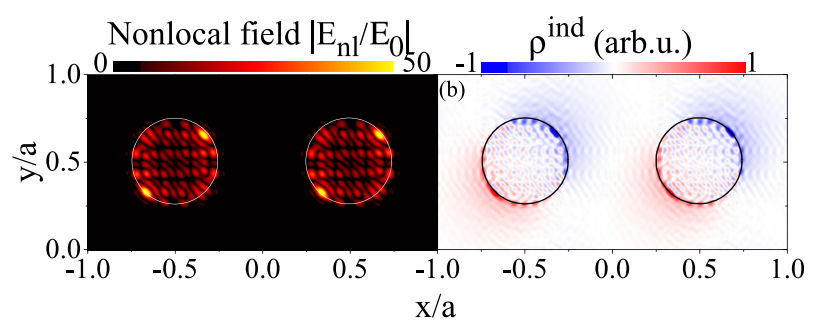

FIG. 6. (a) Contribution of the nonlocal mode $(D=0)$ to the electric field $E_{\mathrm{nl}}$ at the front surface for holey gold films with a lattice constant of $a=200 \mathrm{~nm}$, a radius of $r=0.25 a$, and $t=10 \mathrm{~nm}$ at a resonance frequency of $\lambda=719 \mathrm{~nm}$. (b) Induced charge density for this case. Note that $k_{x}=k_{y}=10^{-4} 2 \pi / a$. results in stronger induced shifts (real part) and an increased broadening (imaginary part) of the considered resonances. This nonlocal increase in the linewidth of plasmon resonances limits the figure of merit (FOM) in nanoplasmonic sensors based on thin metal films patterned with hole arrays.

The surprisingly large structural parameters at which nonlocal effects become sizable can offer a different route to experimentally access this nonlocal regime. We observe induced blueshifts of the resonance position for film thicknesses $t \lesssim 100 \mathrm{~nm}$, impacting also reflectance and absorbance at normal incidence. Hereby, the additional longitudinal waves can yield an effective coupling that reduces both absorption and reflection, thus enhancing further the observed EOT signal. We have demonstrated up to $\pm 6 \%$ relative change in transmittance compared to the local result at the resonance position and an increase of $33 \%$ under off-resonant conditions; other geometries might yield stronger modulations.

The emergence (or disappearance) of absorbance peaks and the observation of Fano-type resonances further indicate that the nonlocal optical response in holey metal films goes beyond the typically observed resonance shifts and plasmon broadening. We have observed the Fano-type resonances only for the extremest case of the diffusion coefficient in GNOR and only for the specific geometry of $a=400 \mathrm{~nm}$ and $r / a=0.25$, and this result needs further studies which are out of scope of the present manuscript. Additional resonances in the optical response contrasting the predictions in the LRA might be a route to verify the presence of nonlocal modes in large-scale, two-dimensional systems.

See supplementary material for the theoretical framework and the dependence on the angle of incidence and the dielectric filling material in the holes in the presence of nonlocal optical response.

C.D. thanks the Deutsche Forschungsgemeinschaft (German Research Foundation) for financial support through a DFG research fellowship (Grant No. 268910011). J.C. gratefully acknowledges financial support from the MINECO through a Ramón y Cajal grant (RYC-2015-17156). The authors thank N. Asger Mortensen for fruitful discussions.

${ }^{1}$ T. W. Ebbesen, H. J. Lezec, H. F. Ghaemi, T. Thio, and P. A. Wolff, Nature 391, 667 (1998).

${ }^{2}$ E. Popov, M. Neviere, S. Enoch, and R. Reinisch, Phys. Rev. B 62, 16100 (2000).

${ }^{3}$ L. Martín-Moreno, F. J. García-Vidal, H. J. Lezec, K. M. Pellerin, T. Thio, J. B. Pendry, and T. W. Ebbesen, Phys. Rev. Lett. 86, 1114 (2001).

${ }^{4}$ Q. Cao and P. Lalanne, Phys. Rev. Lett. 88, 057403 (2002).

${ }^{5}$ M. Beruete, M. Sorolla, I. Campillo, J. S. Dolado, L. Martín-Moreno, J. Bravo-Abad, and F. J. García-Vidal, Opt. Lett. 29, 2500 (2004).

${ }^{6}$ M. Beruete, M. Sorolla, and I. Campillo, Opt. Express 14, 5445 (2006).

${ }^{7}$ F. J. García de Abajo, Rev. Mod. Phys. 79, 1267 (2007).

${ }^{8}$ H. T. Liu and P. Lalanne, Nature 452, 728 (2008).

${ }^{9}$ S. G. Rodrigo, F. J. García-Vidal, and L. Martín-Moreno, Phys. Rev. B 77, 075401 (2008).

${ }^{10}$ F. J. García-Vidal, L. Martín-Moreno, T. W. Ebbesen, and L. Kuipers, Rev. Mod. Phys. 82, 729 (2010).

${ }^{11}$ R. Gordon, D. Sinton, K. L. Kavanagh, and A. G. Brolo, Acc. Chem. Res. 41, 1049 (2008).

${ }^{12}$ C. Genet and T. W. Ebbesen, Nature 445, 39 (2007).

${ }^{13}$ A. Kristensen, J. K. W. Yang, S. I. Bozhevolnyi, S. Link, P. Nordlander, N. J. Halas, and N. A. Mortensen, Nat. Rev. Mater. 2, 16088 (2016). 
${ }^{14}$ N. Yu, J. Fan, Q. J. Wang, C. Pflugl, L. Diehl, T. Edamura, M. Yamanishi, H. Kan, and F. Capasso, Nat. Photonics 2, 564 (2008).

${ }^{15}$ S. I. Bozhevolnyi and N. A. Mortensen, Nanophotonics (published online, 2017).

${ }^{16}$ W. L. Barnes, A. Dereux, and T. Ebbesen, Nature 424, 824 (2003).

${ }^{17}$ J. W. Menezes, J. Ferreira, M. J. L. Santos, L. Cescato, and A. G. Brolo, Adv. Funct. Mater. 20, 3918 (2010).

${ }^{18}$ T. Sannomiya, O. Scholder, K. Jefimovs, C. Hafner, and A. B. Dahlin, Small 7, 1653 (2011).

${ }^{19}$ N. C. Lindquist, P. Nagpal, K. M. McPeak, D. J. Norris, and S.-H. Oh, Rep. Prog. Phys. 75, 036501 (2012).

${ }^{20}$ J. A. Scholl, A. L. Koh, and J. A. Dionne, Nature 483, 421 (2012).

${ }^{21}$ C. Ciracì, R. T. Hill, J. J. Mock, Y. Urzhumov, A. I. FernándezDomínguez, S. A. Maier, J. B. Pendry, A. Chilkoti, and D. R. Smith, Science 337, 1072 (2012).

${ }^{22}$ K. J. Savage, M. M. Hawkeye, R. Esteban, A. G. Borisov, J. Aizpurua, and J. J. Baumberg, Nature 491, 574 (2012).

${ }^{23}$ S. Raza, N. Stenger, S. Kadkhodazadeh, S. V. Fischer, N. Kostesha, A.-P. Jauho, A. Burrows, M. Wubs, and N. A. Mortensen, Nanophotonics 2, 131 (2013).

${ }^{24}$ S. Raza, S. Kadkhodazadeh, T. Christensen, M. Di Vece, M. Wubs, N. A. Mortensen, and N. Stenger, Nat. Commun. 6, 8788 (2015).

${ }^{25}$ A. Varas, P. García-González, J. Feist, F. J. García-Vidal, and A. Rubio, Nanophotonics 5, 409 (2016).

${ }^{26}$ J. B. Lassiter, J. Aizpurua, L. I. Hernandez, D. W. Brandl, I. Romero, S. Lal, J. H. Hafner, P. Nordlander, and N. J. Halas, Nano Lett. 8, 1212 (2008).

${ }^{27}$ J. Zuloaga, E. Prodan, and P. Nordlander, Nano Lett. 9, 887 (2009).

${ }^{28}$ D. C. Marinica, A. K. Kazansky, P. Nordlander, J. Aizpurua, and A. G. Borisov, Nano Lett. 12, 1333 (2012).

${ }^{29}$ R. Esteban, A. G. Borisov, P. Nordlander, and J. Aizpurua, Nat. Commun. 3, 825 (2012).
${ }^{30}$ C. David and F. J. García de Abajo, J. Phys. Chem. C 115, 19470 (2011).

${ }^{31}$ C. David and F. J. García de Abajo, ACS Nano 8, 9558 (2014).

${ }^{32}$ S. Raza, S. I. Bozhevolnyi, M. Wubs, and N. A. Mortensen, J. Phys.: Condens. Matter. 27, 183204 (2015).

${ }^{33}$ C. David, J. Christensen, and N. A. Mortensen, Phys. Rev. B 94, 165410 (2016).

${ }^{34}$ S. Raza, G. Toscano, A.-P. Jauho, M. Wubs, and N. A. Mortensen, Phys. Rev. B 84, 121412(R) (2011).

${ }^{35}$ T. Christensen, W. Yan, S. Raza, A.-P. Jauho, N. A. Mortensen, and M. Wubs, ACS Nano 8, 1745 (2014).

${ }^{36}$ C. David, N. A. Mortensen, and J. Christensen, Sci. Rep. 3, 2526 (2013).

${ }^{37}$ S. Raza, T. Christensen, M. Wubs, S. I. Bozhevolnyi, and N. A. Mortensen, Phys. Rev. B 88, 115401 (2013).

${ }^{38}$ V. Yannopapas, J. Phys.: Condens. Matter 20, 325211 (2008).

${ }^{39}$ W. Yan, M. Wubs, and N. A. Mortensen, Phys. Rev. B 86, 205429 (2012).

${ }^{40} \mathrm{~W}$. Yan, N. A. Mortensen, and M. Wubs, Opt. Express 21, 15026 (2013).

${ }^{41}$ A. Moreau, C. Ciracì, and D. R. Smith, Phys. Rev. B 87, 045401 (2013).

${ }^{42}$ M. Dechaux, P.-H. Tichit, C. Ciracì, J. Benedicto, R. Pollès, E. Centeno, D. R. Smith, and A. Moreau, Phys. Rev. B 93, 045413 (2016).

${ }^{43}$ P. B. Johnson and R. W. Christy, Phys. Rev. B 6, 4370 (1972).

${ }^{44}$ P. Lalanne and G. M. Morris, J. Opt. Soc. Am. A 13, 779-784 (1996).

${ }^{45}$ V. Liu and S. Fan, Comput. Phys. Commun. 183, 2233 (2012).

${ }^{46}$ Longitudinal waves decouple from the magnetic field and thus two separate eigenvalue equations are found.

${ }^{47}$ Note that we use Gauss units throughout this work.

${ }^{48}$ N. A. Mortensen, S. Raza, M. Wubs, T. Søndergaard, and S. I. Bozhevolnyi, Nat. Commun. 5, 3809 (2014).

${ }^{49}$ S. A. Ramakrishna, P. Mandal, K. Jeyadheepan, N. Shukla, S. Chakrabarti, M. Kadic, S. Enoch, and S. Guenneau, Phys. Rev. B 84, 245424 (2011). 